\title{
Effect of the Monomer Ratio on the Strengthening of Polymer Phase Boundaries by Random Copolymers
}

\author{
Chi-An Dai, ${ }^{\dagger}$ Chinedum O. Osuji, ${ }^{\ddagger}$ Klaus D. J andt, ${ }^{\S}$ Benita J . Dair, ${ }^{\ddagger}$ \\ Christopher K. Ober, and E dward J . Kramer*,l
}

Department of Materials Science and Enginering and the Materials Science Center, Cornell University, Ithaca, New York 14853

\section{Chung-Yuen Hui}

Department of Theoretical and Applied Mechanics and the Materials Science Center, Cornell University, Ithaca, New Y ork 14853

Received April 11, 1996; Revised Manuscript Received J uly 1, $1997^{\otimes}$

\begin{abstract}
The fracture toughness $\mathrm{G}_{\mathrm{c}}$ of an interface between the immiscible homopolymers polystyrene (PS) and poly(2-vinyl pyridine) (PVP) reinforced with random copolymers, $\mathrm{dPS}_{\mathrm{f}}-\mathrm{r}-\mathrm{PVP}_{1-f}$ was measured as a function of the average monomer fraction $f$ and the areal chain density $\Sigma$ of the copolymer. Long symmetric random copolymers $(f \approx 0.48$ ) are shown to be effective in strengthening the interfaces. The effectiveness of the random copolymer at low areal chain densities results from each chain establishing multiple covalent connections across the interface. Whether these connections result from each copolymer chain crossing the interface multiple times, entangling with the homopolymer on either side of the interface, or whether these connections result from a "pairing" of chains with different monomer fractions $\mathrm{f}$ (resulting from composition drift) is not yet certain. The interfacial $\mathrm{G}_{\mathrm{c}}$ increases strongly with increasing $\Sigma$ above $\Sigma^{*} \approx 0.004$ chains $/ \mathrm{nm}^{2}$ where a transition from chain scission to crazing occurs. At a high areal density $\left(\Sigma>\Sigma_{\text {sat }}\right.$, where $\Sigma_{\text {sat }}$ is the saturation areal density of the copolymer, above which the random copolymer forms a distinct and continuous layer at the interface), the fracture toughness of the interface reinforced with $f=0.48$ random copolymer becomes a constant. The effectiveness of the copolymer at high $\Sigma$ may be due to the presence within the random copolymers of significant fractions of chains with $f>0.48$ as well as $f<0.48$. Such a spread in composition is caused by composition drift during the bulk copolymerization. In a thick layer of such a copolymer at the interface, the dPS-rich chains will preferentially segregate to the PS/random copolymer interface while the PVP-rich chains will preferentially segregate to the random copolymer/PVP interface, resulting in an overall interface that is graded in composition and highly entangled. For asymmetric random copolymers $(f=0.77,0.60,0.39,0.25)$, the effectiveness decreases markedly as the copolymer becomes less entangled with the homopolymer (corresponding to the minor component in the copolymer) on one side of the interface. The maximum $\mathrm{G}_{\mathrm{c}}$ for the interface saturated with the random copolymer decreases significantly as $f$ deviates from 0.5.
\end{abstract}

\section{Introduction}

The adhesion of polymer interfaces is an issue of technol ogical importance, with applications in polymer alloys, film lamination and coatings, polymer processing, and packaging of semiconductor devices. However, chemically different polymers are normally not miscible due to very low entropy of mixing between such large molecules. Interfaces between immiscible homopolymers are normally very weak but may be markedly strengthened by adding small amounts of a diblock copolymer. ${ }^{1-7}$ By segregating to the interface, diblock copolymers can be used as polymeric surfactants to decrease the interfacial tension between two homopolymer phases.8,9 Diblock copolymers have also been shown to organize themsel ves at the interface ${ }^{2}$ to form molecular "reinforcing connectors" with each block well

\footnotetext{
* To whom correspondence should be addressed.

† Present address: Materials Science and Engineering Division, Manufacturing Research and Engineering Organization, Eastman Kodak Company, Rochester, NY 14652-3701.

$\neq$ Present address: Department of Materials Science and Engineering, Massachusetts Institute of Technology, Cambridge, MA 02139.

$\S$ Present address: Department of Oral and Dental Science, Oralmaterials and Dentalmaterials Section, Bristol University, Bristol, United Kingdom.

"Present address: Departments of Materials and Chemical Engineering, 1361C Engineering II, University of California at Santa Barbara, Santa Barbara, CA 93106.

${ }_{\otimes}$ Abstract published in Advance ACS Abstracts, September 1 , 1997.
}

anchored by entanglements on each side of the interface, thus allowing stress to be transferred across the interface.

In practice, however, long di bl ock copolymers have a number of disadvantages as reinforcing agents. The polymerization methods needed to produce diblock copolymers are usually more expensive than standard free-radical polymerization. In addition, diblock copolymers have a strong tendency to form micelles in one or the other immiscible polymers of the polymer alloy, thus drastically slowing the kinetics of their segregation to interfaces and even producing different "equilibrium" segregation, depending on which polymer phase the diblock copolymer is added to. ${ }^{8-10}$ While segregation ${ }^{8-10}$ and mechanical testing experiments ${ }^{1-7}$ have been done on block copolymer systems, only a few papers have examined the effect of random copolymers.4,11-13 In this study, we show that random copolymers are an attractive alternative to block copolymers for reinforcing polymer interfaces.

Brown et al. 4,5 recently showed that a long random copolymer, $\mathrm{PS}_{\mathrm{f}}-\mathrm{r}-\mathrm{PMMA} \mathrm{A}_{1-\mathrm{f}}$ (PS = polystyrene; $\mathrm{PMMA}$ $=$ poly (methyl methacrylate); $f=0.7$ ) was surprisingly effective in reinforcing PS/PMMA interfaces. Although their results show that the maximum interfacial fracture energy $G_{c}=80 \mathrm{~J} / \mathrm{m}^{2}$ for the random copolymer is smaller than that of their best results for long PS-bPMMA block copolymers $\left(G_{c}=330 \mathrm{~J} / \mathrm{m}^{2}\right)$, the discovery that a random copolymer can be used to enhance the adhesion of polymer interfaces was remarkable. Fur- 
Table 1. Characteristics of the Random Copolymers, dPS $\mathbf{f}-\mathbf{P}-\mathbf{P P}_{\mathbf{1}-\mathbf{f}}$

\begin{tabular}{ccc}
\hline $\begin{array}{c}\text { composition } \\
\text { ratiof }\end{array}$ & $\begin{array}{c}\text { degree of } \\
\text { polymerization } \mathrm{N}_{\mathrm{n}}\end{array}$ & $\begin{array}{c}\text { polydispersity } \\
\text { PDI }\end{array}$ \\
\hline 0.48 & 7000 & 2.5 \\
0.60 & 4200 & 2.7 \\
0.77 & 6700 & 2.5 \\
0.39 & 5640 & 2.32 \\
0.25 & 4440 & 2.5
\end{tabular}

thermore, they proposed that the random copolymer organizes itself so as to make multiple excursions across the interface. Such an organization, which finds some theoretical support in the recent literature, ${ }^{14,15}$ should be disfavored by a value of the PS fraction $f$ that differs significantly from 0.5 . We have tested Brown's hypothesis by measuring the reinforcing effects of long random copolymers $\mathrm{dPS}_{\mathrm{f}}-\mathrm{r}-\mathrm{PVP}_{1-\mathrm{f}}$ at the PS/PVP interface where $\mathrm{f}$ was varied from 0.25 to 0.77 . We show that both the fracture toughness of the interface and the number of monomers per effective crossing of the interface of the random copolymer depend markedly on monomer fraction $f$. A brief report of some of these results was published previously, ${ }^{11}$ but the present paper provides a much more complete picture of random copolymer reinforcement.

\section{Experimental Section}

1. Materials. PS and PVP homopolymers were purchased from Aldrich Chemical Co. and Polysciences, Inc., respectively, and had weight-average molecular weights of $270000\left(\overline{\mathrm{M}}_{\mathrm{w}} / \mathrm{M}_{\mathrm{n}}\right.$ $\approx 2.1)$ and $250000\left(\bar{M}_{\mathrm{w}} / \overline{\mathrm{M}}_{\mathrm{n}} \approx 2.4\right)$, respectively. $\mathrm{PS}$ and $\mathrm{PVP}$ have approximately the same gl ass transition temperature $\left(T_{g}\right.$ $\sim 105^{\circ} \mathrm{C}$ ). Thus, the residual stress upon cooling from the mol ten state at the PS/PVP interface can be minimized. Both homopol ymers deform predominately by crazing; however, PS has a smaller crazing stress than PVP $(\approx 55 \mathrm{MPa}$ for PS and $\approx 75 \mathrm{MPa}$ for PVP). ${ }^{3}$ The deuterated polystyrene and poly(2vinylpyridine) random copolymers, $\mathrm{dPS}_{\mathrm{f}}-\mathrm{r}-\mathrm{PVP}_{1-\mathrm{f}}$ used in this study were synthesized by free-radical bulk polymerization using azobis(isobutyronitrile) (AIBN) as the initiator. The polymerization reaction was carried out in an argon atmosphere at $\sim 70{ }^{\circ} \mathrm{C}$ for 2 days. The characteristics of dPS $\mathrm{S}_{\mathrm{f}}-\mathrm{r}-$ $\mathrm{PVP}_{1-f}$ random copolymers are shown in Table 1 . The molecular weight and composition of these random copolymers were measured by gel permeation chromatography (GPC) and forward recoil spectrometry (FRES). ${ }^{16,17}$ For $f>0.5$, the random copolymer is dPS-rich, and for $f<0.5$, the random copolymer is PVP-rich. It should be noted that $f$ denotes the average styrene fraction in a random copolymer chain. Since styrene and 2-vinylpyridine have different tendencies to undergo copolymerization, there is a composition drift between random copolymer chains that are made at different conversion percentages of the monomers. The reactivity ratios of styrene and 2-vinylpyridine are $\mathrm{r}_{\mathrm{S}} \approx 0.50$ and $\mathrm{r}_{2 \mathrm{VP}} \approx 1.27$, respectively. ${ }^{18}$ These reactivity ratios lead to a tendency for an individual copolymer chain to have more styrene-2-vinylpyridine diads than it would if it were strictly random $\left(r_{s} \times r_{2 V P}\right.$ $=1$ ) and to a tendency for composition drift in bulk copolymerization, with the initial copolymers that form being richer in 2-vinylpyridine than the average composition and the last copolymers that form being richer in styrene. A quantitative assessment of the drift for these different average compositions is shown in the Appendix. Although the reactivity ratios for deuterated styrene and 2-vinyl pyridine have not been reported, we assume that these are similar to those of styrene and $2 \mathrm{VP}$ and thus that the composition drift for our $\mathrm{dPS}_{\mathrm{f}}-\mathrm{r}-\mathrm{PVP}_{1-\mathrm{f}}$ is similar to that reported in the Appendix. The absence of significant blockiness of styrene and 2-vinyl pyridine monomers in the copolymers was verified by proton and ${ }^{13} \mathrm{C} N \mathrm{NMR}$. Detailed ${ }^{13} \mathrm{C}$ NMR spectra are shown in the Appendix. The styrene unit in the copol ymer was deuterated in order to allow for its use as a label in the subsequent FRES experiments. ${ }^{16,17}$

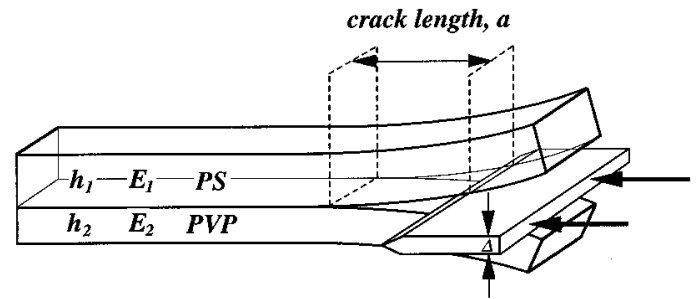

Figure 1. Schematic of the asymmetric double cantilever beam sample for the fracture toughness test.

The FRES technique utilizes a ${ }^{4} \mathrm{He}$ ion beam impinging on an area $\sim 5 \mathrm{~mm} \times 1 \mathrm{~mm}$ of each fracture surface of the sample. The energy of recoiled hydrogen and deuterium particles is recorded and converted into a depth scale based on the energy loss of the particles on their path through the sample. The amount of deuterium averaged over the probe area on each fracture surface is determined by integrating its spectrum signal in FRES, which is readily converted into the areal density of dPS units on each side of the fracture. The total areal chain density of the copolymer can then be found by summing the dPS units on each surface using knowledge of the degree of polymerization of the copolymer and the monomer ratio $f$. By analyzing the ratio of deuterium on the PS side of the fracture to the total deuterium, we also gain important information about the fracture mechanism.

2. Sample Preparation. Plates of polydisperse PS and PVP with dimensions of $5 \mathrm{~cm} \times 7 \mathrm{~cm} \times 2.4 \mathrm{~mm}$ and $5 \mathrm{~cm} \times 7$ $\mathrm{cm} \times 1.7 \mathrm{~mm}$, respectively, were made by compression molding at $160^{\circ} \mathrm{C}$. A thin film of the random copolymer was spun cast from a toluene solution onto the PVP plate. The thickness of the copolymer film and thus the areal chain density $\Sigma$ of the copolymer are controlled by the concentration of the copolymer solution that is spun onto the PVP plate. The homopolymer PS plate is welded to the copolymer-coated PVP plate to form a PS/copolymer/PVP assembly by annealing at $160^{\circ} \mathrm{C}$ for $2 \mathrm{~h}$. The final PS/copolymer/PVP sandwiches were cut into eight strips for the fracture toughness measurements.

3. Fracture Toughness Measurement. The interfacial fracture toughness, or the critical energy release rate, $G_{c}$ is measured using the asymmetric double cantilever beam method (ADCB). A schematic drawing of this method is shown in Figure 1. A razor blade of known thickness $\Delta$ is inserted into the PS/PVP interface. A crack is initiated ahead of the edge of the razor blade. This razor blade is then driven into the interface by a servo motor at a constant speed $\left(3 \times 10^{-6}\right.$ $\mathrm{m} / \mathrm{s}$ ). Steady-state crack propagation was established after several minutes. The entire history of crack progression, in particular the length a of the crack ahead of the razor blade, is recorded using a video camera so that many measurements of $a$, and then $G_{c}$, could be made using one PS/copolymer/PVP strip. The error bars reported subsequently for $G_{c}$ represent 1 standard deviation of at least 16 crack length measurements. The crack length $a$ is converted to $G_{c}$ using the relation first devel oped by Kanninen ${ }^{19}$ and later modified by Creton et al., ${ }^{3}$ i.e.

$$
G_{c}=\frac{3 \Delta^{2}}{8 a^{4}}\left(E_{1} E_{2} h_{1}{ }^{3} h_{2}{ }^{3}\right) \frac{\left(C_{1}{ }^{2} E_{2} h_{2}{ }^{3}+C_{2}{ }^{2} E_{1} h_{1}{ }^{3}\right)}{\left(C_{1}{ }^{3} E_{2} h_{2}{ }^{3}+C_{2}{ }^{3} E_{1} h_{1}\right)^{2}}
$$

where $C_{1}=1+0.64 \mathrm{~h}_{1} / \mathrm{a}$ and $\mathrm{C}_{2}=1+0.64 \mathrm{~h}_{2} / \mathrm{a} . \mathrm{E}_{1}$ and $\mathrm{E}_{2}$ are Young's moduli, and $h_{1}$ and $h_{2}$ are the thicknesses of PS and PVP homopolymer beams, respectively. Occasionally, the crack propagates at the interface in a "stick-slip" fashion. The origin of this stick-slip movement is currently not clear. For most samples only $G_{c}$ values corresponding to stable crack growth are reported. For one sample $(f=0.39)$ stick-slip was so pervasive that average $G_{c}$ values were obtained by measuring the crack length every $30 \mathrm{~s}$ over several cycles of stickslip crack movement. The error bars reported for this composition reflect the large variation in $\mathrm{G}_{\mathrm{c}}$ when stick-slip crack propagation occurs. 
It should be noted that as a crack propagates along an interface between two materials with different el astic moduli, the stress field of the crack tip along the interface in general has both tensile (mode I) and shear (mode II) components. A phase angle $\Psi$ is defined as a measure of the relative shear to the opening components. To ensure the crack propagates at the PS/PVP interface, the plate of more compliant material (PS) is made thicker than the PVP plate, giving rise to a mechanical phase angle $\Psi \approx-6^{\circ}$. At this value of $\Psi$ the fracture toughness of the interface is a minimum and is only weakly dependent on $\Psi$ as long as $\Psi<0$. A detailed discussion of the effects of $\Psi$ on the fracture of PS/PVP interfaces reinforced with diblock copolymers is available el sewhere. ${ }^{20}$

4. TEM Observation of Interface Structure. Crosssectional transmission electron microscopy was used to investigate the structure of the interfaces reinforced with random copolymers. ${ }^{21}$ A small piece of the PS/copolymer/PVP strip was partially embedded in an epoxy resin that was then cured at room temperature so as to provide a sample that would be suitable for mi crotoming. Thin sections ( $100 \mathrm{~nm}$ thick) were cut from the sample strip with a microtome using a glass knife at room temperature. The microtomed film was transferred to the surface of a water bath. Wrinkles in the film induced by the cutting process were removed by exposing the film briefly to a mixed solvent vapor (toluene/chloroform $=50 / 50$ ). This step did not result in noticeable changes in the interfacial structure. The film was subsequently picked up onto a gold grid and dried in vacuum. The film was then exposed to iodine vapor for $12 \mathrm{~h}$ to selectively stain the PVP units. The staining enhances the electron scattering from the PVP units and leads to a distinguishable "darkening" of the PVP-containing phase under TEM. A J EOL 1200EX TEM operating at $120 \mathrm{keV}$ was used for the TEM observations.

\section{Results}

A. Symmetric Random Copolymer, f $\sim 0.5 .1$. Small Areal Density. The fracture toughness $G_{c}$ of the PS/PVP interface reinforced with the symmetric random copolymer $(f=0.48)$ is plotted as a function of its areal chain density in Figure 2a. For $\Sigma<\Sigma_{r}^{*}=$ 0.004 chains $/ \mathrm{nm}^{2}, \mathrm{G}_{\mathrm{c}}$ is low $\left(<5 \mathrm{~J} / \mathrm{m}^{2}\right)$ until a critical value of $\Sigma \approx \Sigma_{r}^{*}=0.004$ chains $/ \mathrm{nm}^{2}$ is exceeded, and then it increases strongly and reaches a value of around $150 \mathrm{~J} / \mathrm{m}^{2}$ for $\Sigma \approx 0.08$ chains $/ \mathrm{nm}^{2}$. It should be noted that the crack sometimes propagates at the interface in a stick-slip fashion. The reported $G_{c}$ values for the $f=0.48$ random copolymer are obtained from measure ments when steady-state crack propagation has been established. The larger $G_{c}$ values for $\Sigma>\Sigma_{r}^{*}=0.004$ chains $/ \mathrm{nm}^{2}$ result from plastic deformation in the form of crazing ahead of the crack tip near the interface region. To establish the presence of crazing, the fracture surfaces were examined using optical mi croscopy. For $\Sigma<\Sigma_{r}^{*}$, the fracture surfaces appeared to be smooth and flat. For $\Sigma>\Sigma_{r}^{*}$ however, the fracture surfaces become rough due to the formation of craze material. To understand the above results, we investigated the fracture mechanism of the interface by measuring the amount of deuterium on each side of the fracture surface. In Figure $2 b$, the fraction of deuterium found on the PS fracture surface is plotted vs $\Sigma$. About $50 \%$ of the deuterium is found on the PS side of the fracture up to a $\Sigma=\Sigma_{r}^{*}=0.004$ chains $/ \mathrm{nm}^{2}$, at which point a transition occurs and the fraction decreases to a value of $\sim 0.2$ for $\Sigma>0.004$ chains $/ \mathrm{nm}^{2}$.

The large increase in the measured $G_{c}$ above the transition as well as the change in the locus of fracture suggests a change in the fracture mechanism with increasing $\Sigma$. For PS/PVP interfaces reinforced with a diblock copolymer 800-870 dPS-b-PVP, Creton et al. ${ }^{3}$
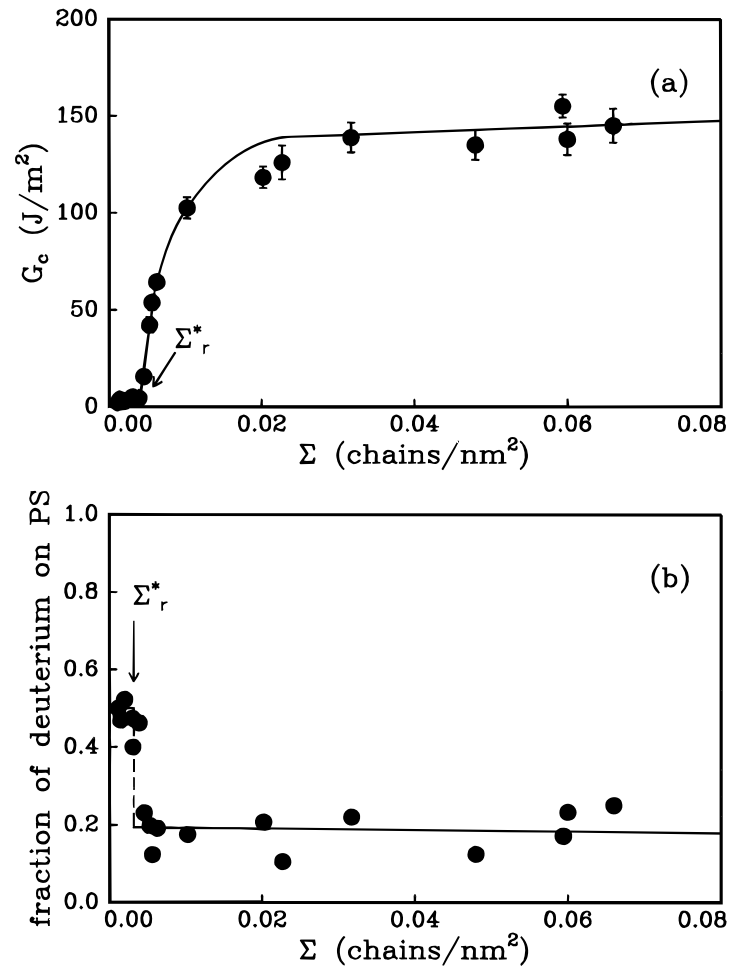

Figure 2. Reinforcement of the PS/PVP interface by a long random copolymer $\mathrm{dPS}_{0.48}-\mathrm{r}-\mathrm{PVP} \mathrm{P}_{0.52}$. (a) Fracture toughness $\mathrm{G}_{\mathrm{c}}$ versus areal chain density $\Sigma$. (b) The fraction of $\mathrm{dPS}$ found on the PS side of the fracture versus areal chain density $\Sigma$.

show that there is a fracture transition from chain scission of the diblock copolymer to crazing of the PS homopolymer on the PS side of the interface above a transition value of $\Sigma_{\mathrm{di}}^{*}=0.03$ chains $/ \mathrm{nm}^{2}$. At the chain scission to crazing transition, the interfacial stress, $\sigma$, is equal to the bond breaking stress of the diblock copolymer $\left(=\mathrm{f}_{\mathrm{b}} \Sigma_{\mathrm{di}}^{*}\right)$ and the crazing stress of the homopolymer PS $\left(=\sigma_{\text {craze }}\right)$ so that

$$
\sigma=\mathrm{f}_{\mathrm{b}} \Sigma_{\mathrm{di}}^{*}=\sigma_{\text {craze }}
$$

where $f_{b}$ is the force to break a single carbon-carbon bond. Equation 2 can be used to estimate $\mathrm{f}_{\mathrm{b}}$ from the measurement of both $\Sigma_{\text {di }}^{*}$ and $\sigma_{\text {craze }}(\approx 55 \mathrm{MPa})$. Creton et al. derived an estimate of $\mathrm{f}_{\mathrm{b}} \approx 2 \times 10^{-9} \mathrm{~N}$ in good agreement with other experimental studies ${ }^{2,22}$ and theoretical predictions. ${ }^{23}$ Creton et al. also demonstrated that there is a change in the locus of fracture upon the failure transition from chain scission to crazing. For $\Sigma<\Sigma_{\text {di }}^{*}=0.03$ chain $/ \mathrm{nm}^{2}$, fracture occurs near the PS/PVP interface and the diblock copolymers are broken near the joint between the dPS and PVP blocks. For $\Sigma>\Sigma_{\mathrm{di}}^{*}=0.03$ chain $/ \mathrm{nm}^{2}$, crazing occurs on the PS side of the interface, followed by failure of the craze fibrils where the dPS block and the homopolymer PS meet. 24,25

Exactly the same kind of fracture transition from chain scission (without crazing) to crazing followed by craze failure is to be expected for the random copolymer. However, the transition areal chain density for the random copolymer $\left(\Sigma_{r}^{*}=0.004\right.$ chains $\left./ \mathrm{nm}^{2}\right)$ is nearly 1 order of magnitude smaller than that of the diblock copolymer $\left(\Sigma_{\text {di }}^{*}=0.03\right.$ chains $\left./ \mathrm{nm}^{2}\right)$. In addition, for $\Sigma<$ $\Sigma_{r}^{*}$ only half the dPS is found on the PS side of the fracture surface for the random copolymer. We defer further discussion of these differences until after presenting the results for the other random copolymers. 

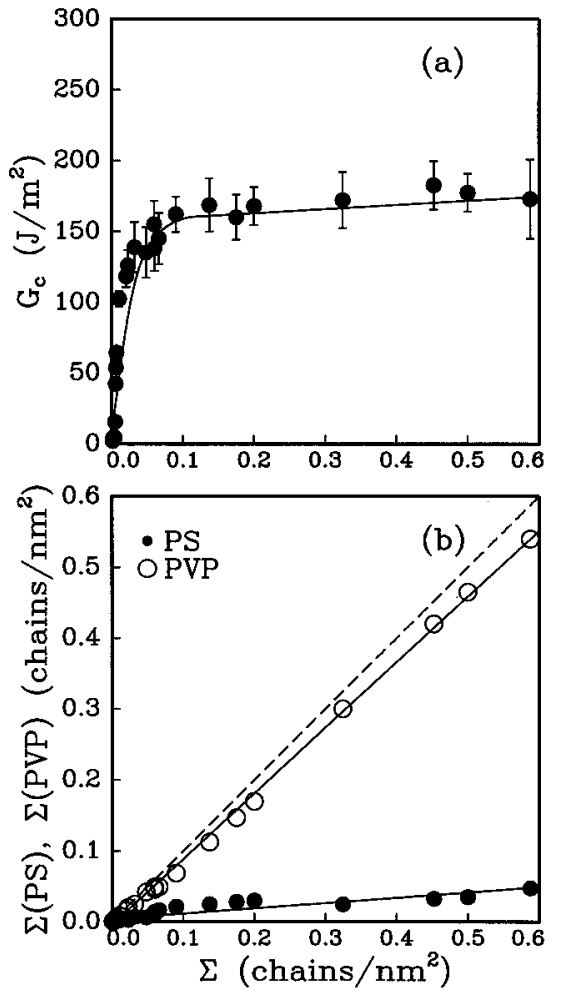

Figure 3. (a) The fracture toughness of the PS/PVP interface reinforced with $\mathrm{dPS}_{0.48}-\mathrm{r}-\mathrm{PVP}_{0.52}$ random copolymer plotted as a function of its areal chain density. (b) Areal chain density of the dPS block found on the PS (0) or PVP (O) side of the fracture surface as a function of $\Sigma$.

\section{$P S$}

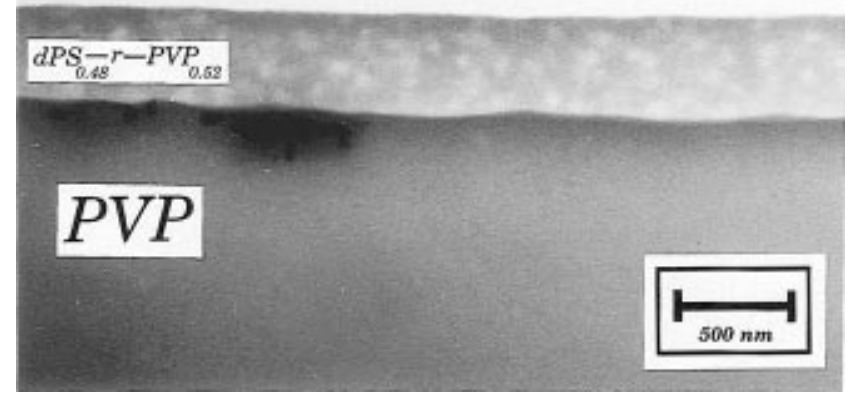

Figure 4. TEM micrograph of a thin film of the PS/PVP interface reinforced with the $\mathrm{dPS}_{0.48}-\mathrm{r}-\mathrm{PVP}_{0.52}$ random copolymer. Due to preferential staining of the PVP phase with iodine, PVP appears to be dark and PS appears to be light. The random copolymer layer has average contrast between that of the PS and PVP homopolymers. The layer thickness of the random copolymer is about $4000 \AA$.

2. Crazing Fracture at High Areal Chain Density. Figure 3a shows $\mathrm{G}_{\mathrm{c}}$ as a function of $\Sigma$ up to $\Sigma \approx$ 0.6 chains $/ \mathrm{nm}^{2}$ for the $\mathrm{dPS}_{0.48-\mathrm{r}-\mathrm{PVP}} \mathrm{P}_{0.52}$ random copolymer. The fracture toughness increases rapidly with increasing $\Sigma$ for $\Sigma \leq 0.08$ chains $/ \mathrm{nm}^{2}$ and then reaches a constant value of around $160 \mathrm{~J} / \mathrm{m}^{2}$ for $\Sigma \geq 0.08$ chains/ $\mathrm{nm}^{2}$. For the highest $\Sigma$ in Figure 3a, our TEM micrograph in Figure 4 shows that the $\mathrm{dPS}_{0.48}-\mathrm{r}-\mathrm{PVP}_{0.52}$ random copolymer forms a distinct layer at the interface. The $\mathrm{dPS}_{0.48}-\mathrm{r}-\mathrm{PVP} \mathrm{P}_{0.52}$ random copol ymer layer has a thickness of $\sim 4000 \AA$, which is well beyond the radius of gyration of the random copolymer chain $(\sim 230 \AA)$.
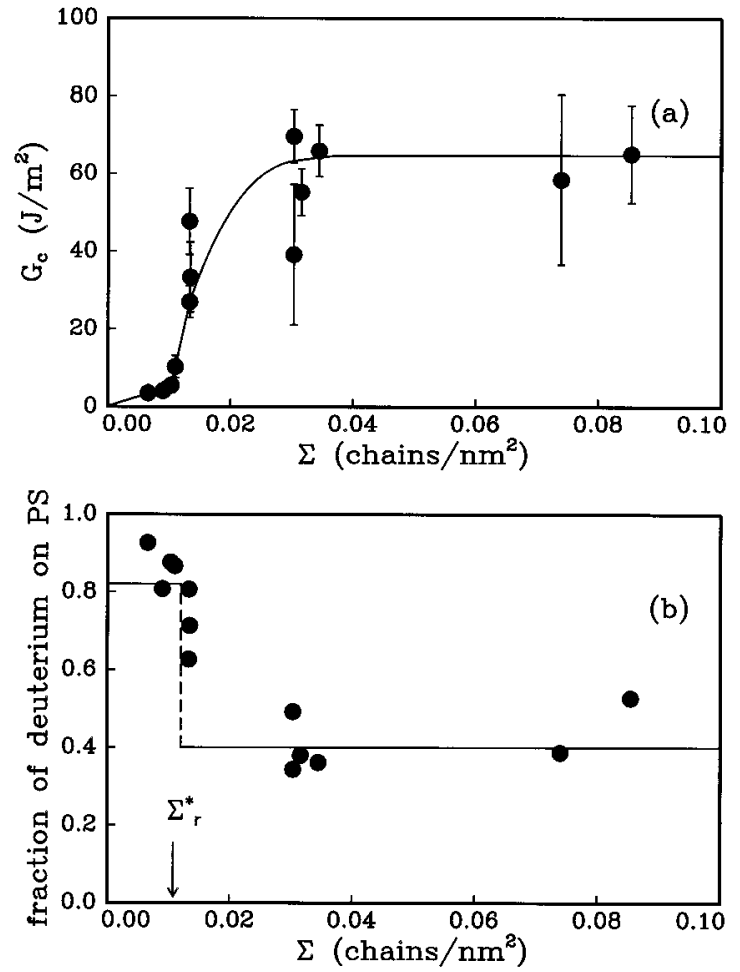

Figure 5. Reinforcement of the PS/PVP interface by a long random copolymer $\mathrm{dPS}_{0.60}-\mathrm{r}-\mathrm{PVP} \mathrm{P}_{0.40}$. (a) Fracture toughness $G_{c}$ versus areal chain density $\Sigma$. (b) The fraction of dPS found on the PS side of the fracture versus areal chain density $\Sigma$.

There does not seem to be any distinct structure within the random copolymer layer, although a mottled staining is observed. In particular, no large-scale redistribution of PVP-rich chains to the PVP interface of the copolymer layer and dPS-rich chains to the PS interface can be seen. This fact, however, does not rule out the local rearrangement of copolymer chains near each interface. In Figure 3b, the apparent areal chain densities of the $\mathrm{dPS}_{0.48}-\mathrm{r}-\mathrm{PVP}_{0.52}$ random copolymer found on the PS or the PVP fracture surface, $\Sigma(P S)$ and $\Sigma(\mathrm{PVP})$, are plotted as a function of $\Sigma$. Note that $\Sigma=$ $\Sigma(\mathrm{PS})+\Sigma(\mathrm{PVP})$. Both plots show that $\Sigma(\mathrm{PVP})$ monotonically increases with increasing $\Sigma(\Sigma(\mathrm{PVP}) \approx \Sigma)$, while $\Sigma(\mathrm{PS})$ remains at very low values $(\sim 0.02-0.05$ chains/ $\mathrm{nm}^{2}$ ) with increasing $\Sigma$. The above result suggests that the locus of fracture is near the interface between the random copolymer layer and the homopolymer PS. The fracture toughness of the interface saturates and remains roughly the same since the crack or craze continues to propagate near the PS/random copolymer interface as soon as the random copolymer layer becomes thick enough to be recognizable as a separate phase.

B. Asymmetric Random Copolymers, $f>0.5$ and $f<0.5$. 1. dPS-Rich (f $>0.5)$ Asymmetric Random Copolymers. The fracture toughness of the interface reinforced with the PS-rich asymmetric random copolymer $(f=0.6)$ is plotted as a function of its areal chain density in Figure 5a. The fracture toughness is low $\left(G_{c}\right.$ $<10 \mathrm{~J} / \mathrm{m}^{2}$ ) for $\Sigma<0.011$ chains $/ \mathrm{nm}^{2}$. $\mathrm{G}_{\mathrm{c}}$ increases strongly for $\Sigma>0.011$ chains $/ \mathrm{nm}^{2}$, and then it reaches a value of around $50 \mathrm{~J} / \mathrm{m}^{2}$ for $\Sigma \approx 0.04$ chains $/ \mathrm{nm}^{2}$. Figure $5 \mathrm{~b}$ shows the fracture of deuterium found on the PS fracture surface as a function of the areal density of the random copolymer ( $f=0.6$ ). For $\Sigma<\sum_{\mathrm{r}, \mathrm{f}=0.6}^{*}=$ 0.011 chains $/ \mathrm{nm}^{2}$, more than $80 \%$ of the deuterium is found on the PS side of the fracture. Above $\Sigma=$ 

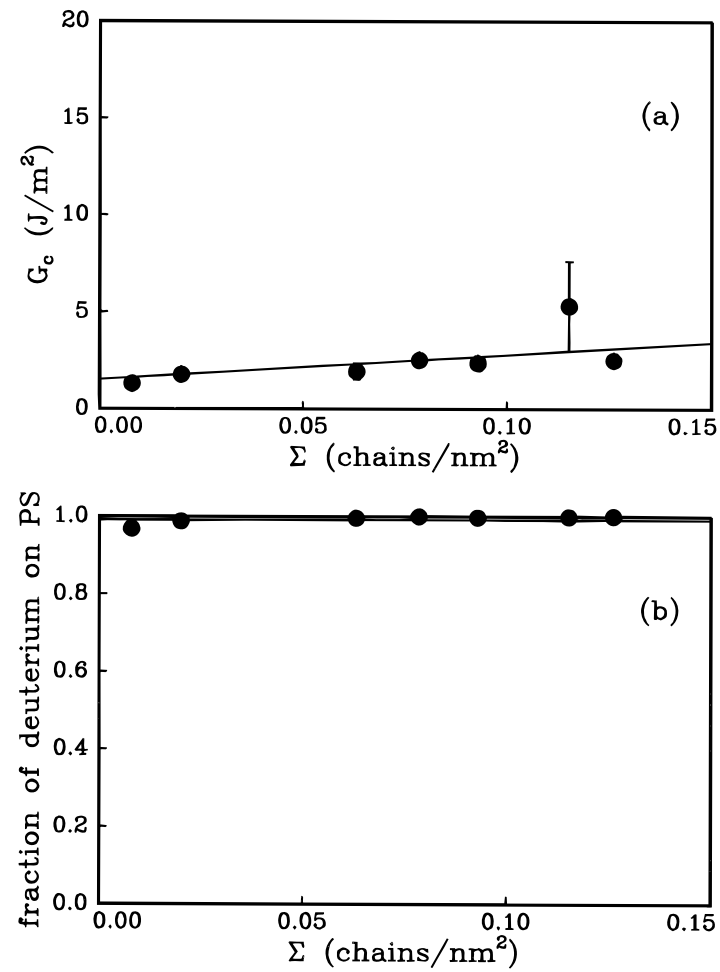

Figure 6. Reinforcement of the PS/PVP interface by a long random copolymer $\mathrm{dPS}_{0.77}-\mathrm{r}-\mathrm{PVP}_{0.23}$. (a) Fracture toughness $G_{c}$ versus areal chain density $\Sigma$. (b) The fraction of dPS found on the PS side of the fracture versus areal chain density $\Sigma$.

$\sum_{\mathrm{r}, \mathrm{f}=0.6}^{*}=0.011$ chains $/ \mathrm{nm}^{2}$, the fraction of deuterium of the PS side is drastically reduced and reaches a value of $\sim 0.4$. The fracture surfaces were also observed using optical microscopy to verify that a wide craze does form on the PS side for $\Sigma>\sum_{\mathrm{r}, \mathrm{f}=0.6}^{*}$ and does not form for $\Sigma<$ $\Sigma_{r, f=0.6}^{*}$. Therefore, a transition from chain scission to crazing followed by craze failure occurs for the $f=0.60$ random copolymer similar to that observed for the $f=$ 0.48 random copolymer. This transition is indicated by a large increase in $\mathrm{G}_{\mathrm{c}}$ and a discontinuous decrease in the deuterium fraction vs $\Sigma$ plot as $\Sigma$ increases above

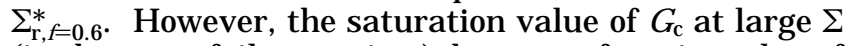
(in the craze failure regime) decreases from its value of $\sim 150 \mathrm{~J} / \mathrm{m}^{2}$ for the $\mathrm{f}=0.48$ copolymer to $\sim 60 \mathrm{~J} / \mathrm{m}^{2}$ for thef $=0.60$ one. A very different situation was observed for the most asymmetric copolymer, $f=0.77$, as shown in Figure $6 a, b$. The fracture toughness of the interface remains very low $\left(G_{c}<5 \mathrm{~J} / \mathrm{m}^{2}\right)$ for all areal densities of the copolymer (up to $\Sigma \approx 0.15$ chains $/ \mathrm{nm}^{2}$ ). Almost all the deuterium ( $\sim 100 \%)$ is found on the PS side of the fracture surface. Optical microscopy of the fracture surfaces shows that a wide craze does not form and the fracture surface remains very smooth. The above result indicates no large-scale plastic deformation (e.g., crazing) occurs for the interface reinforced by the most asymmetric random copolymer $\mathrm{f}=0.77$. The fact that $100 \%$ of the deuterium remains on the PS side suggests that the interface fails by pullout of the copolymer from the PVP side of the interface.

2. PVP-Rich (f $<0.5$ ) Asymmetric Random Copolymers. For the PVP-rich random copolymers ( $f$ $<0.5)$, the results $\left(\mathrm{G}_{\mathrm{c}}\right.$ vs $\Sigma$ and $\Sigma(\mathrm{PS}) / \Sigma$ vs $\Sigma$ ) for $\mathrm{f}=$ 0.39 and for $f=0.25$ are shown in Figures $7 a, b$ and $8 a, b$, respectively. There is a chain scission to crazing transition for the interface reinforced with the $f=0.39$ random copolymer. Notransition is observed, however, for the $f=0.25$ random copolymer. For the $f=0.39$
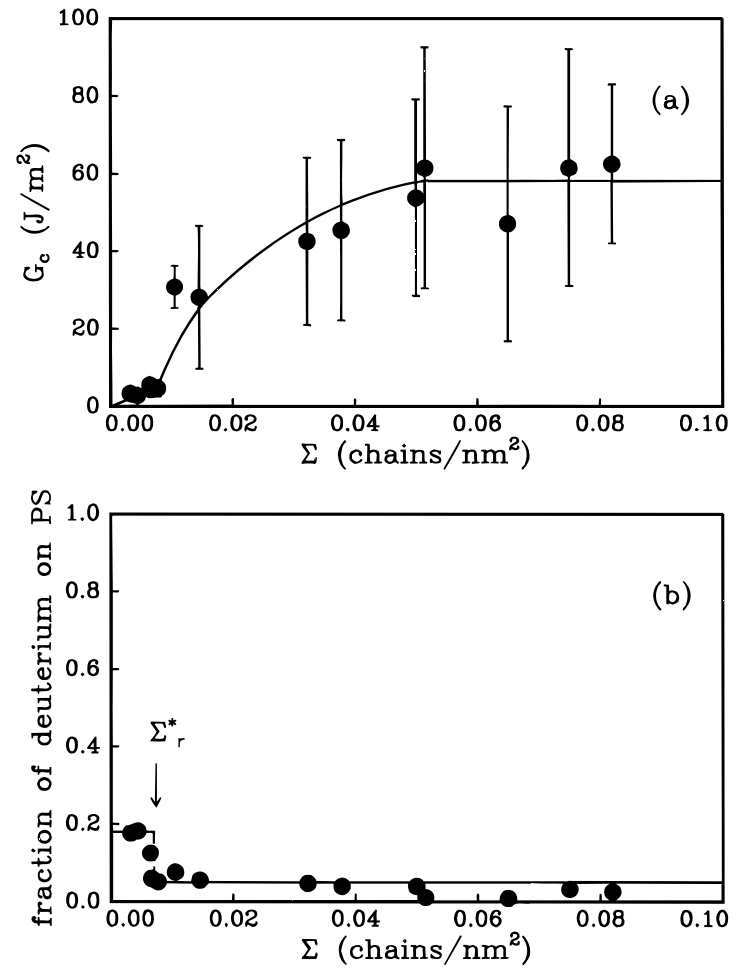

Figure 7. Reinforcement of the PS/PVP interface by a long random copolymer $\mathrm{dPS}_{0.39}-\mathrm{r}-\mathrm{PVP}_{0.61}$. (a) Fracture toughness $\mathrm{G}_{\mathrm{c}}$ versus areal chain density $\Sigma$. (b) The fraction of dPS found on the PS side of the fracture versus areal chain density $\Sigma$.
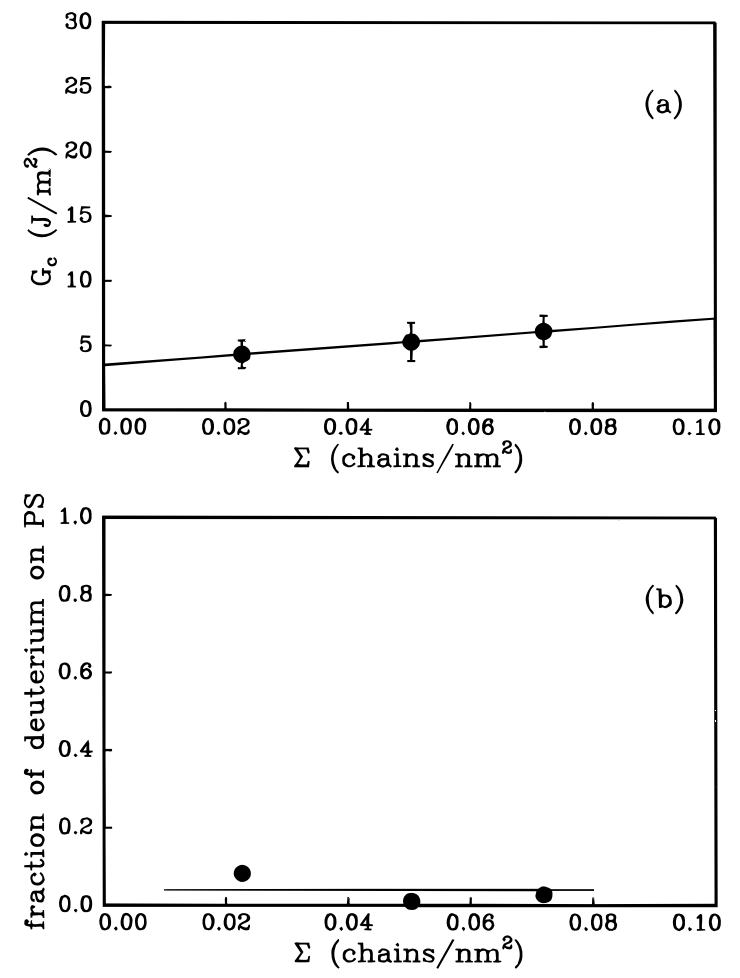

Figure 8. Reinforcement of the PS/PVP interface by a long random copolymer $\mathrm{dPS}_{0.25}-\mathrm{r}-\mathrm{PVP}_{0.75}$. (a) Fracture toughness $G_{c}$ versus areal chain density $\Sigma$. (b) The fraction of $\mathrm{dPS}$ found on the PS side of the fracture versus areal chain density $\Sigma$.

random copolymer, the transition areal chain density, $\Sigma_{\mathrm{r}, \mathrm{f}=0.39}^{*}$ is $\sim 0.009$ chain $/ \mathrm{nm}^{2}$ and the maximum $\mathrm{G}_{\mathrm{c}}$ value at high $\Sigma$ is $\sim 60 \mathrm{~J} / \mathrm{m}^{2}$. The large standard deviation for each fracture toughness measurement is caused in this case by a tendency toward stick-slip crack propagation. For this set of data, the reported 


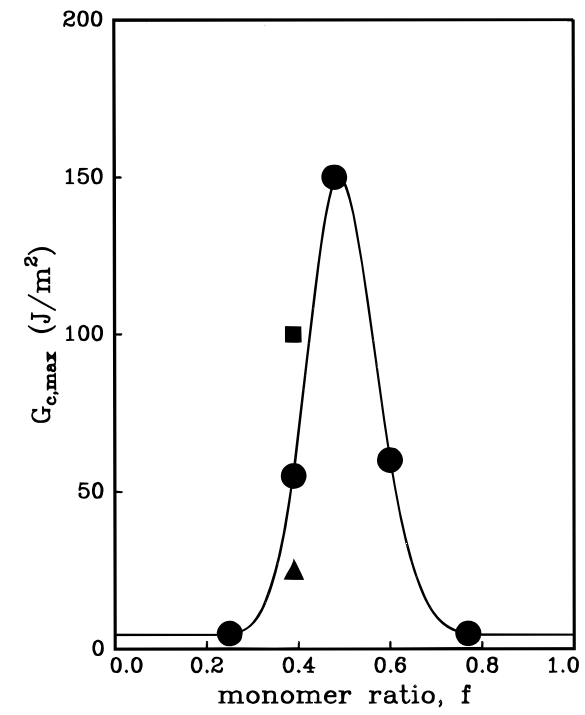

Figure 9. Maximum obtainable fracture toughness, $\mathrm{G}_{c, \max }$, of the interface reinforced with the random copolymers plotted as a function of monomer ratio, $\mathrm{f}$. The $\mathrm{G}_{\mathrm{c}}$ data (solid circle) for the $f=0.39$ random copolymer are reported as the average $\mathrm{G}_{\mathrm{c}}$ value obtained over the stick-slip region. The solid square and solid triangle correspond to the $\mathrm{G}_{\mathrm{c}}$ value as the crack sticks (maximum $G_{c}$ ) and the crack slips (minimum $G_{c}$ ), respectively, for the $f=0.39$ random copolymer. The estimated average $G_{c}$ values for the $f=0.48$ and $f=0.60$ random copolymers are represented by solid circles.

$\mathrm{G}_{\mathrm{c}}$ values are obtained by averaging all $\mathrm{G}_{\mathrm{c}}$ values that are based on the crack length measurements made every $\sim 30$ s over at least one cycle of stick-slip crack propagation. For the $f=0.25$ random copolymer, the $\mathrm{G}_{\mathrm{c}}$ value is always less than $\sim 5 \mathrm{~J} / \mathrm{m}^{2}$ and the fact that nearly $100 \%$ of the deuterium is on the PVP side indicates that the interface fails by pullout of the copolymer from the PS side of the interface.

3. Effect of Copolymer Composition on Maximum F racture Toughness. It is clear from the above results that the monomer ratiof strongly influences the fracture transition and the maximum obtainable fracture toughness of the interface. In Figure 9, the maximum $G_{c}$ observed is plotted as a function of $f$. The $G_{c}$ data (solid circles) for $f=0.48$ and $f=0.60$ random copolymers are reported as the $\mathrm{G}_{\mathrm{c}}$ values corresponding to steady-state propagation. The $\mathrm{G}_{\mathrm{c}}$ data (solid circle) for $f=0.39$, however, is reported as the average $G_{c}$ value obtained over the stick-slip region. The solid square and solid triangle respectively correspond to the $\mathrm{G}_{\mathrm{c}}$ values as the crack sticks (maximum $\mathrm{G}_{\mathrm{c}}$ value) and the crack slips (minimum $G_{c}$ value) for the $f=0.39$ random copolymer. For the other values of $f$ where stick-slip crack propagation occurs, interspersed with steady crack propagation, the "stick" value of $\mathrm{G}_{\mathrm{c}}$ was closest to the steady value so that the solid square is perhaps the data point that can be best compared with the others. In any case as shown in Figure 9, there is a narrow region in $f$ where the random copolymers are effective in reinforcing the polymer interface. The maximum obtainable $G_{c}$ peaks around $f \approx 0.5$ and decreases strongly as $f$ deviates from 0.5 , to either higher or lower values.

\section{Discussion}

There are three apparent regimes of areal chain density of the random copolymer at the interface. At the largest $\Sigma$ 's the random copolymer chains will form a distinct and continuous layer at the interface (regime I). As the areal chain density is decreased, there is an intermediate regi me where the thickness of the random copolymer layer becomes less than the radius of gyration of the random copolymer chains (regime II). The areal chain density $\Sigma^{\Delta}$ at the boundary between regimes I and II can be computed as follows for the symmetric random copolymer ( $f=0.48$ ): Its average radius of gyration, $R_{g}$ $=a \sqrt{\mathrm{N}_{\mathrm{n}} / 6}$, is $\sim 23 \mathrm{~nm}$, where $a$, the statistical segment length, is $\sim 0.67 \mathrm{~nm}$ for both PS and PVP. This boundary areal chain density $\Sigma^{\Delta}$ is given by $R_{g} \rho / N_{n}$, where $\rho$ is the monomer density, $\sim 6$ monomers $/ \mathrm{nm}^{3}$ for PS and PVP; from these numbers $\Sigma^{\Delta} \approx 0.02$ chains $/ \mathrm{nm}^{3}$. Finally at very low areal density (regime III), one can imagine that one of two situations prevails: (i) each random copolymer chain is present at the interface as an isolated free chain without overlapping with other random copolymer chains or (ii) the random copolymers cluster together to form patches of random copolymer of some minimum thickness. One can predict whether case i or case ii obtains by predicting whether twodimensional phase separation of the random copolymer should occur on the interface. In a short paper published previously, 11 we tacitly assumed that case i would obtain in regime III. We will reconsider this assumption below after first discussing the somewhat simpler situation in regime I.

We concentrate on the interface reinforced by the nearly symmetric random copolymer $(f=0.48)$. The failure analysis of this interface in regime I makes it clear that failure occurs al ong the interface between the PS and the random copolymer. The properties of this interface can be discussed in terms of a recent publication by Milner and Fredrickson. ${ }^{27}$ They remind us that the effective Flory interaction parameter, $\chi_{\text {eff, of }}$ a mixture between a random copolymer $A_{f}-B_{1-f}$ and $a$ homopolymer $\mathrm{A}$ can be formulated as follows: ${ }^{28-30}$

$$
\chi_{\text {eff }} \sim(1-f)^{2} \chi_{\mathrm{AB}}
$$

where $\chi_{A B}$ is the Flory segment-segment interaction parameter between pure $A$ and $B$ homopolymers. For an interface between the homopolymer PS and the pure $\mathrm{dPS}_{0.48}-\mathrm{r}-\mathrm{PVP}_{0.52}$ random copolymer layer, $\chi_{\text {eff }}=(1-$ $0.48)^{2} \times 0.12=0.0324$, which is close to the $\chi_{\mathrm{AB}}$ value $(\sim 0.037)$ between the PS and PMMA homopolymers at a similar annealing temperature. ${ }^{26,31}$ However, the fracture toughness of the PS/PMMA interface is relatively small ${ }^{4}\left(G_{c} \sim 10 \mathrm{~J} / \mathrm{m}^{2}\right)$ in contrast to the value we measure for fracture of the symmetric random copolymer/ PS interface $\left(\sim 160 \mathrm{~J} / \mathrm{m}^{2}\right)$ at high $\Sigma$. Based on the thermodynamics of the PS-PVP interaction, our random copolymer reinforced interfaces should be weak.

This discrepancy between theory and experiment is probably caused by the composition drift during the synthesis of the random copolymer. This drift results in the random copolymer being richer in PVP than the average composition at low conversions and leaner in PVP at high conversions. As shown in the Appendix, for a monomer ratio of 0.5 the first $10 \%$ of monomer consumed produces chains of composition $\sim 0.4 \mathrm{dPS}$ while the last $10 \%$ of monomer consumed produces chains of greater than $0.65 \mathrm{dPS}$. Both types of chains, as well as those with intermediate $f$, exist in the final copolymer. The dPS-rich chains may segregate at the random copolymer/PS interface, while the PVP-rich chains may segregate at the PVP/random copolymer interface. Self-consistent-field simulations of blends of copolymers of different $f$ show, in fact, that this preferential segregation is to be expected. ${ }^{32}$ Assuming that 
the random copolymer at the PS interface has $f>0.65$ means that the $\chi$ eff for that interface is less than 0.015 . Such an interface would have an interfacial width $\sim$ $2 \mathrm{a} /\left(6 \chi_{\text {eff }}\right)^{1 / 2}$ of greater than $45 \AA$ compared to a predicted interface width of $\sim 30 \AA$ for an interface between PS and a $\mathrm{dPS}_{0.48}-\mathrm{r}-\mathrm{PVP}_{0.52}$ random copolymer with no composition drift. The broader interface would result in a better entanglement between the PS and the random copolymer layer and thus a larger fracture toughness.

This hypothesis can allow us also to understand the decrease in fracture toughness of the PS/PVP interfaces reinforced with the asymmetric random copolymers as $\mathrm{f}$ departs from its symmetric value of 0.5 . F or an $\mathrm{f}=$ 0.75 copolymer the first $10 \%$ of monomer consumed produces chains of the smallest styrene content, $f \approx$ 0.65 . The $\chi_{\text {eff }}$ of the interface between that composition and the PVP will be $\sim 0.05$, larger than that for PS/ PMMA. The fact that the $f=0.77$ copolymer reinforced interface fails in regime I by pullout at the PVP/ copolymer boundary is thus not surprising. Similarly, for the $f=0.25$ copolymer the last $10 \%$ of monomer consumed produces copolymer chains with $f \approx 0.35$. The interface between that composition and PS also has a $\chi_{\text {eff }}$ of 0.05 . This interface should then fail at the boundary between the PS and the copolymer layer as observed.

If these ideas are correct, then a broadened composition range in the random copolymer would lead to an increase in fracture toughness. To test this consequence of the hypothesis, we have made 50-50 mixtures of pairs of the asymmetric random copolymer; i.e., one mixture was made by combining the $f=0.60$ random copolymer with the $f=0.39$ random copolymer and a second by combining the $f=0.77$ copolymer with the $f$ $=0.25$ copolymer. While the detailed results on these mixtures will be reported elsewhere, we note that the $\mathrm{G}_{\mathrm{c}}$ of an interface reinforced with a thick layer (thickness $\gg R_{g}$ ) of the $0.60 / 0.39$ mixture is $\sim 150 \mathrm{~J} / \mathrm{m}^{2}$, approximately the same as the $G_{c}$ of the interface reinforced by a similarly thick layer of the $f=0.48$ random copolymer. This value is much larger than that of interfaces reinforced by either the $f=0.60$ or the $f=$ 0.39 random copolymers $\left(\mathrm{G}_{\mathrm{c}} \approx 60 \mathrm{~J} / \mathrm{m}^{2}\right)$. Even more surprising is the fact that the $G_{c}$ of an interface reinforced with a thick layer of the $0.77 / 0.25 \mathrm{mixture}$ is 100 J $/ \mathrm{m}^{2}$ and shows clear evidence of crazing prior to fracture. From the amount of deuterium on the PS side of these interfaces it is clear that the craze fails somewhere near the center of the random copolymer mixture layer and not at either boundary between it and the homopolymers. These results thus strongly support our hypothesis that the composition drift during the polymerization of the random copolymer can lead to an increase in fracturetoughness of the interface reinforced by that random copolymer. H owever, to prove definitely that the composition drift contributes to the effectiveness of the $\mathrm{f}=0.48$ random copolymer in strengthening the interface, one needs to synthesize a random copolymer of the same overall composition but with a narrow composition range. Such a copolymer can be made, in principle, by controlling the conversion of the monomer or the mole fraction in the monomer feed. Experiments to produce such copolymers are underway currently.

We consider now the boundary between regimes I and II. The fraction of deuterium on the PS side shown in Figure $2 b$ demonstrates that final fracture of the craze occurs along the interface between the $f=0.48$ random

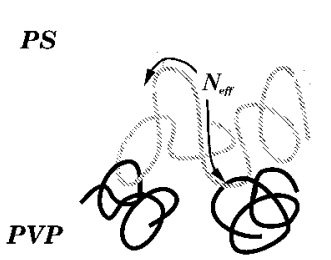

(a)

(b)

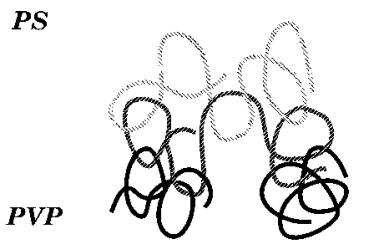

Figure 10. (a) Schematic illustration of the organization at the interface of the random copolymer, which has $\mathrm{N}_{\text {eff }}$ monomer units per effective crossing of the interface. (b) Schematic illustration of the organization of the random copol ymers with different monomer fractions that form a cluster at the interface.

copolymer chains and the homopolymer PS in the crazing regime $\left(\Sigma>\Sigma_{r}^{*}\right)$. The fracture toughness increases with increasing areal chain density of the random copolymer until the $\mathrm{G}_{\mathrm{c}}$ value saturates for $\Sigma \geq$ 0.02 chain $/ \mathrm{nm}^{2}$. The areal density above which $\mathrm{G}_{c}$ saturates should correspond to the boundary areal density, $\Sigma^{\Delta}$, where the random copolymer has formed a fully overlapped thin film layer. The fact that our estimate of $\Sigma^{\Delta} \approx 0.02$ chains $/ \mathrm{nm}^{2}$ agrees with the areal chain density above which the fracture toughness saturates is thus not surprising.

Finally, we consider the situation at the lowest areal chain densities. A clear transition is observed between chain scission and crazing for the $f=0.48$ as well as the $f=0.39$ and $f=0.60$ copolymers. The fact that $\Sigma_{r}^{*}$ occurs at much lower areal chain density than the similar transition for the diblock copolymer reinforced interface, $\Sigma_{\mathrm{di}}^{*} \approx 0.03$ chains $/ \mathrm{nm}^{2}$, must mean that somehow each random copolymer chain establishes multiple covalent connections across the interface. Since the stress to break the random copolymer chain $=f_{b} \sum n_{\text {cross }}$, where $n_{\text {cross }}$ is the average number of connections per chain that are established across the interface and where $f_{b}$ is the force to break a single carbon-carbon bond, the criterion for the transition from chain scission to crazing followed by craze failure becomes

$$
\Sigma_{\mathrm{r}}^{*}=\sigma_{\text {cross }} / \mathrm{n}_{\text {cross }} \mathrm{f}_{\mathrm{b}}
$$

Equations 2 and 4 imply that $\Sigma_{r}^{*}$ and $\Sigma_{\text {di }}^{*}$ are related by

$$
\Sigma_{\mathrm{r}}^{*}=\Sigma_{\mathrm{di}}^{*} / \mathrm{n}_{\text {cross }}
$$

Using this result, one can define an average number of monomer units per effective connection, $\mathbf{N}_{\text {eff, }}$ as equal to $\mathrm{N}_{\mathrm{n}} / \mathrm{n}_{\text {cross, }}$ where $\mathrm{N}_{\mathrm{n}}$ is the degree of polymerization of the random copolymer chain. We can therefore estimate the average number of effective connections per random copolymer chain to be eight, or one about every $\mathrm{N}_{\text {eff }}=$ 900 units of the $f=0.48$ random copolymer chain.

One random copolymer chain morphology that might give rise to this behavior is shown in Figure 10a. This figure shows schematically an isolated random copolymer weaving back and forth across the PS/PVP interface, forming with each effective crossing entangled 
loops (or ends) in both the PS and the PVP phases. The fact that such loops might occur was suggested by recent theoretical treatments of an isol ated random copolymer at a fluid/fluid interface. ${ }^{14,15}$ There are two problems with this isolated chain picture however. The first is that if we assume that random copolymer has its random-coil radius of gyration of about $230 \AA$, a set of such coils placed uniformly along the interface begin to overlap at $\Sigma \approx 0.002$ chains $/ \mathrm{nm}^{2}$, well below $\Sigma_{r}^{*} \approx 0.004$ chains $/ \mathrm{nm}^{2}$. The second problem is that Milner and Fredrickson ${ }^{27}$ have shown that the random-coil reference state used for isol ated chain calculations ${ }^{14,15}$ is not appropriate. They demonstrate that such random copolymer coils should be collapsed, both either in homopolymer and presumably then also at the interface. Under conditions where the chains are collapsed at the interface, the radius of such coils would be about $65 \AA$ so that these would not overlap if they were distributed uniformly. The catch is that these collapsed coils would strongly attract each other and would form a twodimensional phase-separated microstructure at the interface consisting of pancake regions of copolymer separated by regions of bare interface. Recent Monte Carlo simulations show that such pancake clusters are to be expected. 33 Using the arguments of Milner and Fredrickson, it is easy to show that these copolymer patches wet the interface; i.e., they have zero macroscopic contact angle and thus will not form spherical droplets. The patches will have an edge energy that will tend to cause them to coarsen, but it seems likely that a thin film of copolymer deposited at the interface will break up into a 2D microstructure on a scale not too much larger than the uniform film thickness and that because of the very high molecular weight of the copolymer, the coarsening of this microstructure will be very slow.

Thus, a more realistic molecular picture might be the one shown in Figure 10b, which shows two copolymer molecules of different composition in a copol ymer patch. The dPS-rich molecule penetrates into the PS phase to form entangl ements, while the PVP-rich molecule penetrates and forms entanglements with the PVP homopolymer. Each molecule separately would have few if any molecular connections to the phase corresponding to its minor component. But because the copolymer molecules with these different compositions can entangle with each other, molecular bridges are formed from the PS to the PVP phase. Because the molecules are so long, there is more than one bridge formed per molecule and thus the decrease in $\Sigma_{r}^{*}$ can be explained. In the chain scission regime the fracture of such a patch would lead to about $50 \%$ of the deuterium on each side of the fracture in agreement with our observations for the $f=0.48$ copolymer. In regards to the chain scission to crazing transition, it is important to note that stress at the interface to cause crazing must occur over an area corresponding to at least several craze fibrils, of order $100 \mathrm{~nm}$. If the copolymer patches at the interface are much smaller than these dimensions, as seems likely, the stress for crazing would be that averaged over many copolymer patches and bare PS/PVP interface regions. Thus, the areal chain density of copolymer, which controls the area fraction of such patches, is still the controlling parameter for the transition from chain scission to crazing, as observed.

\section{Conclusions}

We have measured the fracture toughness of the interface reinforced with random copolymers as a func- tion of average monomer fraction $f$ and the areal chain density $\Sigma$ of the copolymer. The results show that long, symmetric, random copolymers can be remarkably effective in reinforcing immiscible polymer interfaces. We have demonstrated that the effectiveness of the random copolymer decreases significantly as the average monomer ratio, $\mathrm{f}$, deviates from 0.5 . The high fracture toughness of the $f=0.48$ copolymer is likely to be due to the composition drift during the polymerization of the random copolymer. Thus, the use of random copolymers with an even broader range of composition may provide a new method for strengthening the interfaces between two immiscible homopolymers.

Acknowledgment. We acknowledge the support of the Cornell Materials Science Center which is funded by the NSF-DMR-MRSEC program and fellowship support from the Dow Chemical Co. through the Polymer Outreach Program at Cornell. K.D.J . is grateful for partial financial support in the form of a FeodorLynen Fellowship of the Alexander von Humboldt Foundation, Bonn, Germany. The authors also thank Lynn Jelinski for the NMR experiments and Anna Balazs and Glenn Fredrickson for their helpful discussion.

\section{Appendix}

1. Composition Drift. Since the reactivity ratios of styrene (S) and 2-vinylpyridine (2VP) are different $\left(r_{S} \approx 0.50, r_{2 V P} \approx 1.27\right),{ }^{18}$ styrene and 2-vinylpyridine have different tendencies to undergo copolymerization. This fact can lead to a variation of composition in the copolymer chains produced from a reaction batch where the composition of the monomers continuously changes as the copolymerization reaction proceeds. A simple calculation can be made to estimate the degree of this composition drift. In Figure 11a, the styrene fraction in the copolymer chain is plotted as a function of monomer conversion for an initial composition of $50 \%$ of styrene and $50 \%$ of 2VP. The composition of styrene in the chains that form initially is lower than $50 \%$ ( $\sim 40 \%$ ) for low conversion since styrene has a lower tendency to be added to the growing chain. However, as more 2VP monomers are consumed, more styrenes are added to the copolymer chain at higher conversion. At high conversion, e.g., 95\%, the styrene fraction has a value of $\sim 0.8$, which is considerably larger than the average composition ratio, $f$, of $\sim 0.5$. The random copolymer with high styrene fracture is expected to be more miscible with the homopolymer PS. However, more than $90 \%$ of the copolymer chains that are copolymerized has a styrene fraction between 0.4 and 0.6. For an initial composition in the feed of $75 \%$ of styrene (25\% of 2VP) and of $25 \%$ of styrene $(75 \%$ of 2VP), the plots for the composition drift are shown in parts $b$ and $c$ of Figure 11 , respectively. For $75 \%$ of styrene in the initial feed, the styrene fraction is in the range of $0.6-1.0$. F or $25 \%$ of styrene in the initial feed, the styrene fraction is in the range of $0.2-0.4$.

2. NMR Measurement. The noise-decoupled ${ }^{13} \mathrm{C}$ NMR spectra were recorded using a Varian 300XL spectrometer at $75 \mathrm{MHz}$. The samples were analyzed as $10-15 \%(\mathrm{w} / \mathrm{v})$ solution in perdeuterated $\mathrm{N}, \mathrm{N}$-dimethylformamide (DMF $-d_{7}$ ) at $130^{\circ} \mathrm{C}$ using TMS as the internal standard. For comparison, both homopolymer PVP and symmetric random copolymer made by the same synthesis procedure are used. The assignment of the different signals to various carbon atoms in the monomer unit was made by comparing each spectrum 

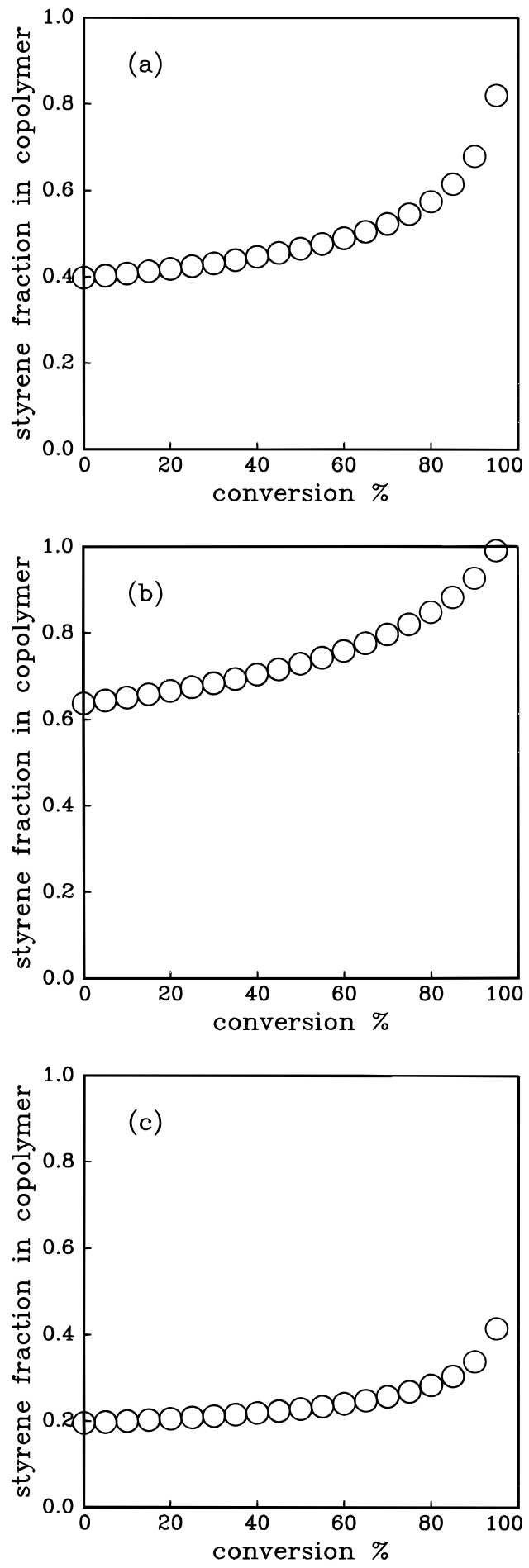

Figure 11. Styrene fraction in the copolymer chain plotted as a function of the extent of copolymerization (conversion) for an initial composition of (a) $50 \%$ of styrene and $50 \%$ of 2-vinylpyridine, (b) $75 \%$ of styrene and $25 \%$ of 2 -vinylpyridine, and (c) $25 \%$ of styrene and $75 \%$ of 2 -vinylpyridine in a batch.

with that listed in ref 34 . Figures 12 and 13 show the NMR spectra of $C_{1}$ in the pyridine ring for the PVP

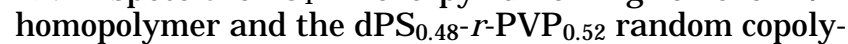
mer, respectively. The signals from $\mathrm{C}_{1}$ in the PVP homopolymer are split into two main peaks with several finer peaks due to the tacticity effects. This result suggests this carbon position is sensitive to the structural arrangement of the monomer unit. However, for the symmetric random copol ymer the finer structure of the signal of $C_{1}$ disappears, presumably as a result of the random insertion of styrene units into the chain.

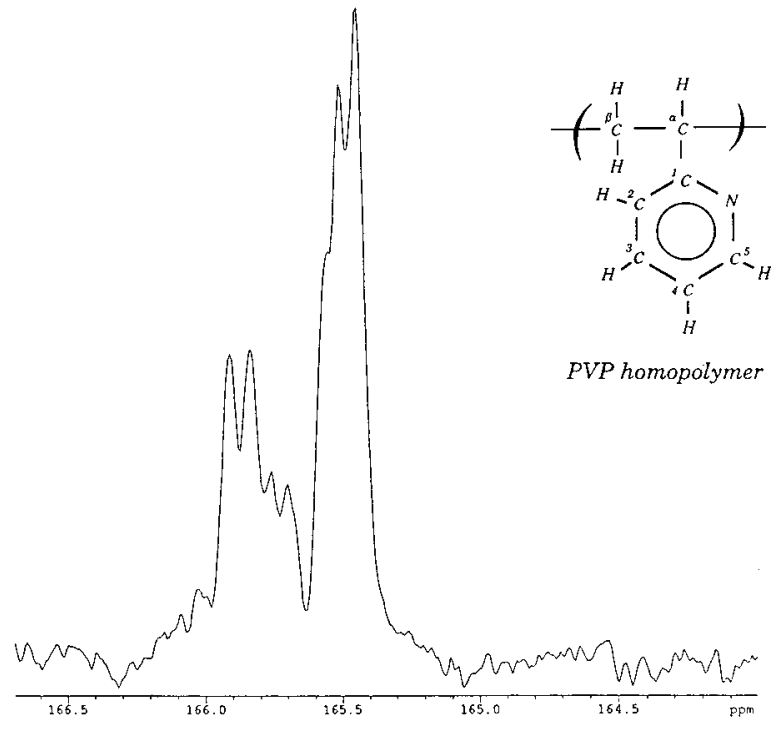

Figure 12. Expanded carbon-13 NMR spectrum of the $C_{1}$ resonance of the PVP homopolymer observed in DMF- $d_{7}$ at $130{ }^{\circ} \mathrm{C}$.

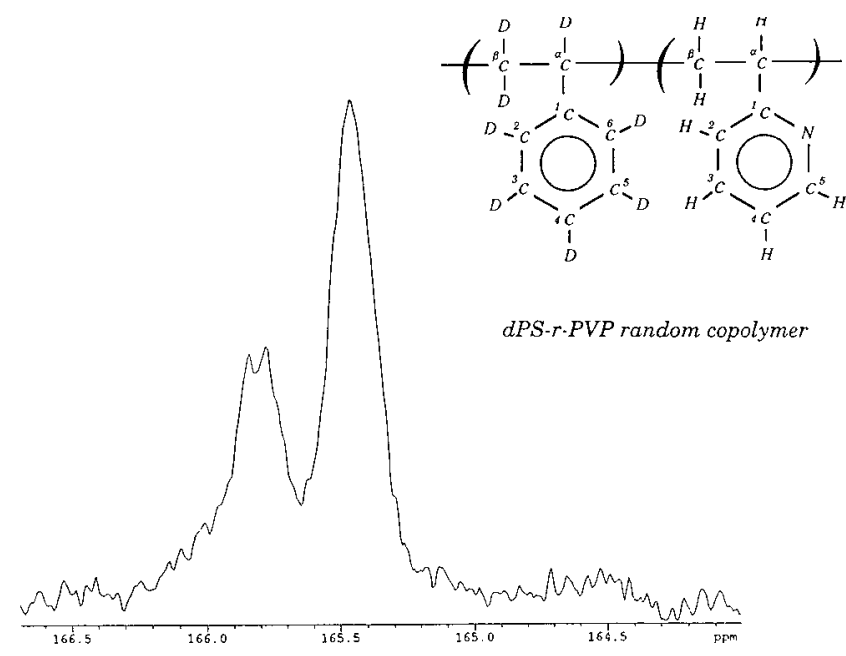

Figure 13. Expanded carbon-13 NMR spectrum of the $C_{1}$ resonance of the $\mathrm{dPS}_{0.48}-\mathrm{r}-\mathrm{PVP}_{0.52}$ random copol ymer in DMF $\mathrm{d}_{7}$ at $130^{\circ} \mathrm{C}$.

Although there is limited information on the assignment of each peak with the tacticity of the homopolymer chain, the result indicates that the random copolymer cannot be too "blocky". H owever, a quantitative determination of whether there are small deviations from randomness of the monomer sequence distribution of the random copolymer chain cannot be made based on these NMR data.

\section{References and Notes}

(1) Brown, H. R. Macromolecules 1989, 22, 2859.

(2) Brown, H. R.; Deline, V. R.; Green, P. F. Nature (London) 1989, 341, 221.

(3) Creton, C. F.; Kramer, E. J .; Hui, C.-Y.; Brown, H. R. Macromol ecules 1992, 25, 3075.

(4) Brown, H. R.; Char, K.; Deline, V. R.; Green, P. F. Macromolecules 1993, 26, 4155.

(5) Char, K.; Brown, H. R.; Deline, V. R. Macromolecules 1993 26, 4164.

(6) Washiyama, J .; Creton, C. F.; Kramer, E. J .; Xiao, F.; Hui, C.-Y. Macromol ecules 1993, 26, 6011.

(7) Creton, C. F.; Brown, H. R.; Deline, V. R. Macromolecules 1994, 27, 1774.

(8) Shull, K. R.; Kramer, E. J .; Hadziioannou, G.; Tang, W. Macromol ecules 1990, 23, 4780. 
6736 Dai et al.

(9) Dai, K. H.; Kramer, E. J .; Shull, K. R. Macromolecules 1992, $25,220$.

(10) Hu, W.; Koberstein, J . T.; Lingelser, J . P.; Gallot, Y. Macromolecules 1995, 28, 5209.

(11) Dai, C.-A.; Dair, B. J .; Dai, K. H.; Ober, C. K.; Kramer, E.J .; Hui, C.-Y.; J elinski, L. W. Phys. Rev. Lett. 1994, 73, 2472.

(12) Sikka, M.; Chen, L.; Winey, K. I. Bull. Am. Phys. Soc. 1995, 40, 544.

(13) Kulasekere, R.; Ankner, J. A.; Kaiser, H.; Russell, T. P.; Mayes, A. M. Bull. Am. Phys. Soc. 1995, 40, 612.

(14) Yeung, C.; Balazs, A. C.; J asnow, D. Macromolecules 1992, 24, 1357.

(15) Garel, T.; Huse, D. A.; Leibler, S.; Orland, H. Europhys. Lett. 1989, 8, 9.

(16) Doyle, B. L.; Peercy, P. S. Appl. Phys. Lett. 1979, 34, 811.

(17) Mills, P. J.; Green, P. F.; Palmstrøm, C. J .; Mayer, J. W.; Kramer, E. J. Appl. Phys. Lett. 1984, 45, 957.

(18) Brown, F. E.; Ham, G. E. J . Polym. Sci. 1964, A2, 3623.

(19) Kanninen, M. F. Int. J . Fract. 1973, 9, 83.

(20) Xiao, F.; Hui, C.-Y.; Washiyama, J .; Kramer, E. J . Macromolecules 1994, 27, 4382.

(21) Washiyama, J .; Creton, C.; Kramer, E. J . Macromolecules 1992, 25, 4751.

(22) Odell, J . A.; Keller, A. J . Polym. Sci., Polym. Phys. Ed. 1986, 24, 1889.
(23) Kausch, H. H. Polymer Fracture, 2nd ed.; Springer Verlag: Berlin, 1987.

(24) Brown, H. R. Macromolecules 1991, 24, 2757.

(25) Sha, Y.; Hui, C.-Y.; Ruina, A.; Kramer, E. J . Macromolecules 1995, 28, 2450.

(26) Dai, K. H.; Kramer, E. J . Polymer 1994, 35, 157.

(27) Milner, S. T.; Fredrickson, G. H. Macromolecules 1995, 28, 7953.

(28) Gehlsen, M. D.; Rosedale, J . H.; Bates, F. S.; Wignall, G. D.; Hansen, L.; Almdal, K. Phys. Rev. Lett. 1992, 68, 2452.

(29) ten Brinke, G.; Karasz, F. E.; MacKnight, W. J . Macromolecules 1983, 16, 1827.

(30) Kambour, R. P.; Bendler, J . T.; Bopp, R. C. Macromolecules $1983,16,753$

(31) Russell, T. P.; Hjelm, R. P., J r.; Seeger, P. A. Macromolecules 1990, 23, 890.

(32) Gersappe, D.; Balazs, A. G. Phys. Rev. E 1995, 52, 5061.

(33) Lyatskaya, Y.; Gersappe, D.; Gross, N. A.; Balazs, A. C. J . Phys. Chem. 1996, 100, 1449.

(34) Pham, Q.-T.; Petiaud, R.; Waton, H. Proton and Carbon NMR Spectra of Polymers; J ohn Wiley \& Sons, Inc.: New York, 1983.

MA960543M 